\title{
Aberrant expression of selectin E, CXCL1, and CXCL13 in chronic endometritis
}

\author{
Kotaro Kitaya and Tadahiro Yasuo \\ Department of Obstetrics and Gynecology, Kyoto Prefectural University of Medicine, Kamigyo-ku, Kyoto, \\ Japan
}

\begin{abstract}
Chronic endometritis is often identified in the patients with unexplained infertility, and is histopathologically characterized by infiltration of plasmacytes within the endometrial stroma. In parallel with stromal plasmacyte infiltration, the endometrial functional layer in chronic endometritis is invaded by B cells, which are a rare leukocyte subset residing within the basal layer in the nonpathological endometrium. In this study, we investigated the molecular expression underlying this unusual increase of $B$ cells in chronic endometritis. Twenty-two out of 76 infertile patients were diagnosed with chronic endometritis from the stromal plasmacyte infiltration, and the endometrium contained numerous stromal B-cell aggregates and glandular single B cells. However, the other major leukocyte subsets, including $\mathrm{T}$ cells, natural killer cells, macrophages, and neutrophils were comparable in densities in chronic endometritis and nonpathological endometrium. The microvascular endothelium showed immunoreactivity to adhesion molecule selectin E and chemokine CXCL13 along with immunoreactivity to CXCL1 in the glandular epithelium in chronic endometritis, but not in the nonpathological endometrium. Lipopolysaccharide significantly induced surface selectin $E$ expression and CXCL13 secretion in uterine microvascular endothelial cells, and CXCL1 secretion in endometrial epithelial cells in vitro. These findings indicated that the aberrant local microenvironment triggered possibly by bacterial infection has a role in selective extravasation of circulating $B$ cells in chronic endometritis.

Modern Pathology (2010) 23, 1136-1146; doi:10.1038/modpathol.2010.98; published online 21 May 2010
\end{abstract}

Keywords: B cells; chronic endometritis; CXCL1; CXCL13; selectin E

Endometritis is subdivided into two categories: acute endometritis, which is recognized as microabscess formation or neutrophil invasion within the endometrial glands, and chronic endometritis, which is distinguished by plasmacyte infiltration within the endometrial stroma. ${ }^{1}$ Chronic endometritis is frequently identified in women suffering from repeated implantation failure after in vitro fertilization and transfer of morphologically good embryos, as well as abnormal uterine bleeding and pelvic inflammatory disease. ${ }^{2-4}$ The implantation rates after embryo transfer are significantly lower in the infertile patients with chronic endometritis than in those without chronic endometritis. ${ }^{2}$ Chronic endometritis is often overlooked due to its morphological resemblance to secretory phase endometrium. ${ }^{1}$

Correspondence: Dr K Kitaya, MD, Department of Obstetrics and Gynecology, Kyoto Prefectural University of Medicine, 465 Kajii-cho, Kamigyo-ku, Kyoto 602-8566, Japan.

E-mail: kitaya@koto.kpu-m.ac.jp

Received 29 January 2010; revised 8 March 2010; accepted 12 March 2010; published online 21 May 2010
Plasmacytes are sometimes indistinguishable from endometrial stromal fibroblasts by conventional tissue staining methods and localize so focally that they can be missed in small biopsy specimens. ${ }^{5,6}$

A recent study demonstrated the prominent B-cell invasion into the endometrial functional layer in chronic endometritis. ${ }^{7}$ In the nonpathological endometrium, B cells are a rare leukocyte population that comprises less than $1 \%$ of all endometrial leukocytes. B cells are found mostly in the basal layer, as the core cells of the leukocyte aggregates consisting of surrounding numerous CD8 + T cells and macrophages, but are rarely seen as single cells in the functional layer in the nonpathological endometrium. ${ }^{8,9}$ Although the mechanism underlying the focal accumulation of B cells in chronic endometritis remains unclear, it is conceivable that some aberrant local microenvironment induces the selective extravasation of circulating B cells across uterine microvessels and helps these cells proliferate and differentiate in situ into plasmacytes.

For extravasation, leukocytes are required to undergo serial, organized, multistep interactions 
with endothelial cells. ${ }^{10}$ These sequential processes start with tethering/rolling, a weak bonding of leukocytes to endothelial cells. The next step is reciprocal cell activation triggered by chemokines that are produced mainly in endothelial cells. These early interactions lead leukocytes to the following tight adhesion to endothelial cells and transendothelial migration. Of the known adhesion molecules, the transmembrane glycoproteins mucosal addressin cell adhesion molecule (MAdCAM)-1, selectin $\mathrm{P}$, and selectin $\mathrm{E}$, and the immunoglobulin superfamily member vascular cell adhesion molecule (VCAM)-1 are potentially involved in circulating B-cell tethering/rolling on endothelial cells. MAdCAM-1 is expressed constitutively in high endothelial venules in Peyer's patch and lamina propria, and has a crucial role in tethering/rolling of naive and integrin $\alpha 4 \beta 7+$ memory $\mathrm{B}$ cells. ${ }^{11}$ Selectin E and VCAM-1 are not produced in resting endothelial cells, but are induced upon some activating signals. VCAM-1 contributes to tethering/rolling of integrin $\alpha 4 \beta 7$ - memory $\mathrm{B}$ cells by interaction with very late antigen-4 on $B$ cells, whereas selectin E binds to endoglycan, a sialomucin presented on circulating $\mathrm{B}$ cells. ${ }^{11,12}$ Selectin $\mathrm{P}$ expression is confined to Weibel-Palade bodies in resting endothelial cells and is relocated on the cell surface rapidly after activation serving as another ligand to endoglycan. In addition, circulating B cells show selective migratory responses to the specific chemokines such as CCL-2, -19, -21, CXCL-1, -8, -12, and -13, which are the ligands for CCR-2, -7, CXCR-1, $-2,-4$, or $-5 .^{13-15}$

In the nonpathological endometrium, MAdCAM-1, VCAM-1, CCL-2, -19, -21 and CXCL-8, and -13 are expressed throughout the menstrual cycle or temporally during the secretory phase, whereas selectin E, and CXCL12 are not detectable in any phase in the cycle. ${ }^{16-23}$ The presence of these adhesion molecules and chemokines in the endometrium with chronic endometritis, however, remains undetermined. In this study, we investigated the expression, localization, and potential induction signals of these molecules in chronic endometritis.

\section{Materials and methods}

\section{Antibodies and Reagents}

Ready-to-use mouse monoclonal antibodies against human CD45 (leukocyte common antigen, 2B11), CD3 (T-cell marker, PS1), CD20 (B-cell marker, L26), CD56 (natural killer (NK) cell marker, 1B6), CD68 (macrophage marker, PG-M1), and syndecan-1 (plasmacyte marker, B-A38) ${ }^{24}$ were obtained from Nichirei (Tokyo, Japan). Mouse monoclonal antineutrophil elastase antibody (NP57, 1:50 dilution) was obtained from Dako (Kyoto, Japan). Mouse monoclonal antibodies against human leukocyte antigen-G (invasive trophoblast marker, 4H84, 1:100 dilution), selectin E (P2H3, 1:50 dilution), and VCAM-1 (E-10, 1:50 dilution) were obtained from Santa Cruz Biotechnology (Santa Cruz, CA, USA), as well as goat polyclonal antibodies against CCL19 (C-19, 1:10 dilution), CCL21 (C-15, 1:100 dilution), and MADCAM-1 (K-19, 1:10 dilution). Mouse monoclonal antibodies against CXCL1 (MAB275, 1:50 dilution), CXCL8 (MAB6217, 1:10 dilution), CXCL12 (MAB310, 1:2 dilution), CXCL13 (MAB801, 1:50 dilution), and CCL2 (MAB2791, 1:20 dilution) were obtained from R\&D Systems (Minneapolis, MN, USA). Mouse monoclonal antiselectin P antibody (MCA796T, 1:10 dilution) was obtained from AbD Serotec (Oxford, UK).

\section{Immunohistochemistry}

Endometrial specimens were obtained from 76 patients with unexplained infertility and who had undergone biopsy in search of endometrial pathology on days 6 to 8 after urinary luteinizing hormonesurge detection (Clearplan, Inverness Medical, Tokyo, Japan). Serum estradiol and progesterone concentrations were measured by chemiluminescent immunoassay (intra- and interassay coefficient of variation $<10 \%$; SRL, Tokyo, Japan). The specimens were fixed overnight in $4 \%$ paraformaldehyde (in phosphate buffer (pH 7.3)), embedded in paraffin, and cut into $4 \mu \mathrm{m}$ sections. After being dewaxed in xylene and rehydrated in a graded series of ethanol, the sections were dated according to the standard criteria ${ }^{25}$ and immunostained with $4 \mathrm{H} 84$ to exclude the presence of the invasive trophoblasts. The remaining sections were used for immunohistochemistrical analysis. All patients had given us their informed consent. The study was approved by the Institutional Review Board. The sections were subjected to microwave pretreatment for antigen retrieval in the citrate buffer solution ( $\mathrm{pH}$ 6.0) for $5 \mathrm{~min}$, and immersed in 3\% hydrogen peroxide for 5 min to block endogenous peroxidase. After being washed, the sections were incubated with $10 \%$ fetal calf serum for $10 \mathrm{~min}$ to suppress nonspecific antibody binding. The sections were then incubated with antibody B-A38 or control antibody for 30 min at room temperature. After being washed, the sections were incubated with horseradish peroxidase-conjugated secondary antibody (Dako) for $30 \mathrm{~min}$ at room temperature, washed, and developed with diaminobenzidine. The immunostaining for other antigens was performed by similar protocols. We skipped the antigen retrieval technique for the immunostaining for CD45, adhesion molecules, and chemokines. The immunoreactive cells were enumerated in 10 nonoverlapping stromal areas and 20 glandular lumina under a light microscope $(\times 400$ magnification) by two independent observers who were indifferent to the study. The immunoreactive cells clearly flanked by two epithelial cells and located above the epithelial basement membrane were enumerated per 100 epithelial cells. ${ }^{9}$ 


\section{Culture and Stimulation of Human Uterine Microvascular Endothelial Cells and Endometrial Epithelial Cells}

Human uterine microvascular endothelial cells (Cambrex, Walkersville, MD, USA) were maintained in medium 199 (Invitrogen, Carlsbad, CA, USA) supplemented with $0.03 \mathrm{M}$ HEPES, $10 \%$ charcoalstripped fetal calf serum, and antibiotics $(100 \mathrm{IU} / \mathrm{ml}$ penicillin, $100 \mu \mathrm{g} / \mathrm{ml}$ streptomycin, and $2.5 \mu \mathrm{g} / \mathrm{ml}$ amphotericin B). ${ }^{26}$ After 2-4 passages, the cells $\left(10^{5}\right.$ cells $\left./ \mathrm{ml}\right)$ were cultured with or without $10 \mathrm{ng} / \mathrm{ml}$ recombinant interleukin- $1 \beta$, tumor necrosis factor- $\alpha$, interferon- $\gamma$, or lipopolysaccharide (Peprotech, London, UK) at $37^{\circ} \mathrm{C}$ in humidified $5 \% \quad \mathrm{CO}_{2}$ for $48 \mathrm{~h}$. The cells were scraped, washed, and incubated with $\mathrm{P} 2 \mathrm{H} 3$ or control antibody for $15 \mathrm{~min}$ at room temperature. The cells were washed and then incubated with fluorescein isothiocyanateconjugated secondary antibody (BD Biosciences, San Diego, CA, USA) for $15 \mathrm{~min}$. The cells were washed and then analyzed using a FACS Calibur and CellQuest (BD Biosciences). The culture supernatant was retrieved and frozen at $-80^{\circ} \mathrm{C}$ until measurement. The supernatant CXCL13 concentration was quantitated in triplicate by a Quantikine kit (R\&D Systems). The minimal detectable level was $5 \mathrm{pg} / \mathrm{ml}$.

Endometrial epithelial cells were isolated as previously described. ${ }^{27}$ Nonpathological endometrium $(n=5)$ was minced into $\sim 1 \mathrm{~mm}^{3}$ in Dulbecco's modified Eagle medium (Invitrogen) containing $0.5 \%$ collagenase (Sigma-Aldrich, St Louis, MO, USA) for $1 \mathrm{~h}$ at $37^{\circ} \mathrm{C}$. The cell suspension was then passed through a nylon mesh (BD Biosciences). The endometrial glands retained on the mesh were washed free of adhering stromal cells with Hanks' balanced salt solution, transferred by backwashing with the medium, and seeded on sterile plastic dishes. After allowing the remaining stromal cells to adhere to the dishes for $30 \mathrm{~min}$, the unattached epithelial cells were collected. The cell purity was confirmed by immunostaining with anti-cytokeratin antibody (Dako). After being resuspended in the medium, the cells $\left(10^{6}\right.$ cells $\left./ \mathrm{ml}\right)$ were cultured with or without $10 \mathrm{ng} / \mathrm{ml}$ recombinant cytokines or lipopolysaccharide at $37^{\circ} \mathrm{C}$ in humidified $5 \% \mathrm{CO}_{2}$ for $48 \mathrm{~h}$. The culture supernatant was frozen at $-80^{\circ} \mathrm{C}$ until measurement. The supernatant CXCL1 concentration was quantitated in triplicate by a Quantikine kit (R\&D Systems). The minimal detectable level was $15 \mathrm{pg} / \mathrm{ml}$.

\section{Statistics}

Each data set was analyzed for normal distribution using $\chi^{2}$ goodness-of-fit test. The two groups were compared with Student's $t$-test, nonparametric Mann-Whitney $U$-test, or two-by-two contingency table $\chi^{2}$-independence test. Dunnett's multiple comparison test was used to identify the difference versus the control group. Values with $P<0.05$ were considered as significantly different.

\section{Results}

\section{Marked B-Cell Infiltration into the Endometrium with Chronic Endometritis}

Weak membranous or cytoplasmic immunostaining for syndecan-1 was detected in the surface and glandular epithelium in all endometrial samples examined (Figure 1a and b). Twenty-two cases $(28.9 \%)$ were diagnosed as chronic endometritis by the presence of the punctate immunostaining for syndecan-1 scattered throughout the stroma (Figure 1b). The number of stromal plasmacytes in 10 nonoverlapping stromal areas varied from 12 to 53 (mean 25). According to the dating criteria, seven of the 22 chronic endometritis specimens showed out-ofphase morphology with delay of two or more days. Immunostaining for control antibody (Figure 1g) was not detectable.

In the nonpathological endometrium, the immunostaining for CD20 was detected as a few single cells in the stroma, but was not detectable within the surface epithelium, glandular epithelium, or glandular lumina (Figure 1c). In the chronic endometritis specimens, the immunostaining for CD20 was detected as dense focal stromal aggregates in the functional layer, along with single cells between epithelial cells and within gland lumina (Figure 1d). The stromal B-cell aggregates were often composed of more than 20 cells without clear cell boundaries. The stromal B-cell density was more than 30 in 10 nonoverlapping stromal areas in all chronic endometritis samples examined, while it was always less than four in the samples without chronic endometritis $(P<0.00001)$. The stromal densities of T cells, NK cells (Figure 1e and f), macrophages, and neutrophils in the chronic endometritis were comparable with those in the nonpathological endometrium $(P>0.14)$ (Table 1).

\footnotetext{
Figure 1 Representative microphotographs of immunohistochemistry for leukocyte subset-specific antigens in human endometrium with or without chronic endometritis. The microphotographs represent the immunostaining for syndecan-1 in the nonpathological endometrium (a) and syndecan-1 in chronic endometritis (b), CD20 in the nonpathological endometrium (c) and CD20 in chronic endometritis (d), and CD56 in the nonpathological endometrium (e) and CD56 in chronic endometritis (f). Photo (g) represents immunostaining with control IgG in chronic endometritis. The sections were counterstained with hematoxylin. The arrows indicate the immunostaining with diaminobenzidine. The scale bars indicate $50 \mu \mathrm{m}$.
} 


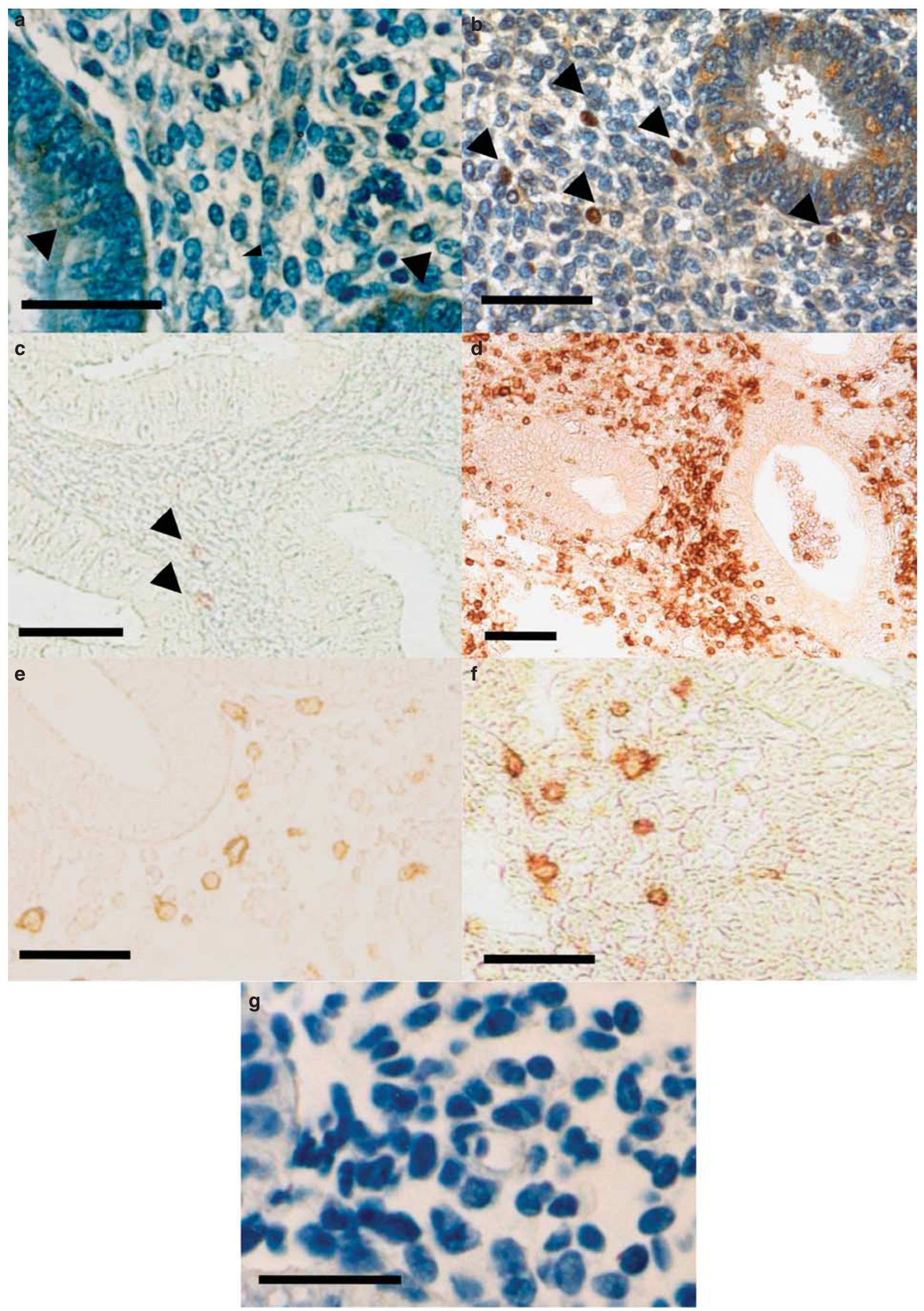




\section{Expression of Selectin E in Microvascular Endothelium in Chronic Endometritis}

In all specimens examined (12 chronic endometritis and 11 nonpathological endometrium), the immunostaining for VCAM-1 (Figure 2a and b) and MAdCAM-1 (Figure 2c and d) was noted in the surface and glandular epithelium, along with faint immunostaining for MADCAM1 in the stroma. Immunostaining for selectin $\mathrm{P}$ and selectin $\mathrm{E}$ was not detectable in the epithelium in any of the specimens examined (Figure 2e-g).

Faint immunostaining for VCAM-1 and MADCAM-1 and distinct immunostaining for selectin $\mathrm{P}$ appeared in the microvascular endothelium in the endometrial functional layer in chronic endometritis as well as the nonpathological endometrium (Figure 2a-e). The proportion of microvessels immunoreactive for VCAM-1 and MAdCAM-1 was comparable $(P>0.42)$ in the nonpathological endometrium (67-100\% for VCAM-1, and $57-100 \%$ for MADCAM-1) and chronic endometritis (62-100\% for VCAM-1, and $57-100 \%$ for MADCAM-1). The endothelial immunostaining for selectin $\mathrm{P}$ was detectable in every identifiable microvessel in all specimens examined (Figure 2e). Conversely, the immunostaining for selectin $\mathrm{E}$ in the endometrial microvascular endothelium in chronic endometritis was strikingly different from that in the nonpatho- logical endometrial specimens. Although the endothelial immunostaining for selectin $\mathrm{E}$ was not detectable in any of the nonpathological endometrium (Figure 2f), it was detected in $78-100 \%$ of microvessels in all chronic endometritis specimens examined (Figure 2g).

\section{Expression of CXCL13 in Microvascular Endothelium and CXCL1 in Epithelium in Chronic Endometritis}

The immunostaining pattern for all CC chemokines examined, CXCL8 and CXCL12, was similar in the chronic endometritis $(n=11)$ and nonpathological endometrium $(n=10)$. The immunostaining for CCL2 was detected faintly in the epithelium but not in the microvascular endothelium (Figure 3a). The immunostaining for CCL19 (figure 3b) and CCL21 (data not shown) was detected in the epithelium and microvascular endothelium. The immunostaining for CCL21 resembled that for CCL19, but the immunostaining in the microvascular endothelium was weaker than that in the epithelium. The immunostaining for CXCL8 was detected in the epithelium along with perivascular stroma (Figure 3c). The immunostaining for CXCL12 was not detectable in any samples examined (data not shown). On the contrary, the immunostaining for CXCL13 was confined to the epithelium in

Table 1 Density of endometrial leukocyte subsets in nonpathological endometrium versus chronic endometritis

\begin{tabular}{|c|c|c|c|}
\hline & Nonpathological endometrium & Chronic endometritis & $\mathrm{P}$-value \\
\hline \multicolumn{4}{|l|}{ Stromal leukocytes } \\
\hline CD20+ (B cells) & $0.41(0-3)$ & $47(32-81)$ & $<0.00001$ \\
\hline CD3+ (T cells $)$ & $17(3-37)$ & $19(4-35)$ & 0.38 \\
\hline CD56+ (NK cells) & $35(1-118)$ & $38(2-131)$ & 0.56 \\
\hline CD68+ (macrophages) & $6.9(1-15.5)$ & $9.5(1.5-33)$ & 0.30 \\
\hline Neutrophil elastase+ (neutrophils) & $3.8(0-11)$ & $5.7(0-17)$ & 0.14 \\
\hline \multicolumn{4}{|l|}{ Intraepithelial leukocytes } \\
\hline CD20+ (B cells) & $0(0-0)$ & $6(2-12)$ & $<0.00001$ \\
\hline CD3+ (T cells $)$ & $0(0-0)$ & $0(0-0)$ & $>0.99$ \\
\hline CD56+ (NK cells) & $0.36(0-2)$ & $0.5(0-2)$ & $>0.99$ \\
\hline CD68+ (macrophages) & $0(0-0)$ & $0(0-0)$ & $>0.99$ \\
\hline Neutrophil elastase+ (neutrophils) & $0(0-0)$ & $0(0-0)$ & $>0.99$ \\
\hline \multicolumn{4}{|l|}{ Glandular luminal leukocytes } \\
\hline CD20+ (B cells) & $0(0-0)$ & $1.5(0-4)$ & $<0.00001$ \\
\hline CD3+ (T cells $)$ & $0(0-0)$ & $0(0-0)$ & $>0.99$ \\
\hline CD56+ (NK cells) & $0.045(0-1)$ & $0.091(0-1)$ & 0.79 \\
\hline CD68+ (macrophages) & $0(0-0)$ & $0(0-0)$ & $>0.99$ \\
\hline Neutrophil elastase+ (neutrophils) & $0(0-0)$ & $0(0-0)$ & $>0.99$ \\
\hline
\end{tabular}

The numbers represent the mean and range (in parentheses) leukocyte counts in 10 nonoverlapping stromal areas, per 100 epithelial cells, or 20 gland lumina.

\footnotetext{
Figure 2 Representative microphotographs of immunohistochemistry for adhesion molecules in human endometrium with or without chronic endometritis. The microphotographs represent the immunostaining for VCAM-1 in the nonpathological endometrium (a) and VCAM-1 in chronic endometritis (b), MAdCAM-1 in the nonpathological endometrium (c) and MAdCAM-1 in chronic endometritis (d), selectin P in chronic endometritis (e), and selectin E in the nonpathological endometrium (f) and selectin E in chronic endometritis (g). The sections were counterstained with hematoxylin. The arrows indicate the immunostaining with diaminobenzidine. The scale bars indicate $50 \mu \mathrm{m}$.
} 

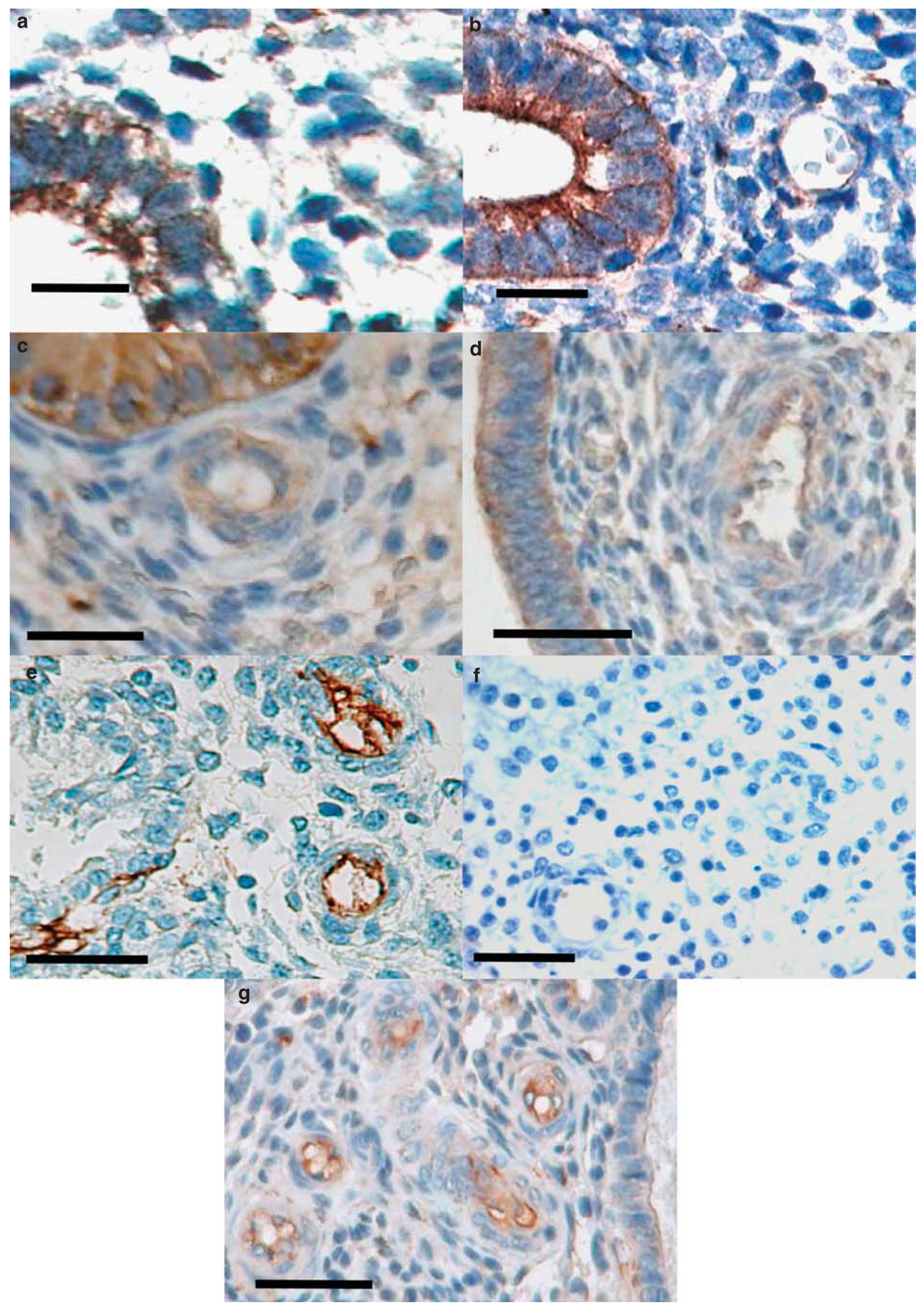
the nonpathological endometrium (Figure 3d), but was detected distinctly both in the epithelium and microvascular endothelium along with some diffuse stromal immunostaining in chronic endometritis specimens (Figure 3e). The proportion of microvessels immunoreactive for CXCL13 in chronic endometritis specimens varied from 43 to $100 \%$. The immunostaining for CXCL1 also showed some differences. The immunostaining for CXCL1 in the stroma was patchy, but not detectable in the epithelium or microvascular endothelium in the nonpathological endometrium (Figure 3f). In contrast, the immunostaining for CXCL1 was distinct in the glandular epithelium with some patchy immunostaining in the stroma in eight $(72.7 \%)$ chronic endometritis specimens, whereas the immunostaining in the microvascular endothelium was faint (Figure 3g).

\section{Lipopolysaccharide Induces CXCL13 Secretion and Selectin E Expression in Human Uterine Microvascular Endothelial Cells in Vitro}

Selectin E was detected on $1.7 \pm 0.4 \%$ (mean and s.d., $n=5$ ) of the unstimulated human uterine microvascular endothelial cells. Interleukin- $1 \beta$, tumor necrosis factor- $\alpha$, and lipopolysaccharide significantly $(P<0.017)$ enhanced the selectin $\mathrm{E}$ expression rate and mean fluorescent intensity ratio (versus control antibody) in five independent experiments (Figure 4), whereas interferon- $\gamma$ did not affect these indexes. The effect of lipopolysaccharide was significantly $(P<0.0040)$ greater than that of interleukin- $1 \beta$ and tumor necrosis factor- $\alpha$.

CXCL13 was not detectable in the culture supernatant of the uterine microvascular endothelial cells after interleukin- $1 \beta$, interferon- $\gamma$ treatment, or control culture in any of five independent experiments (Figure 5a). In contrast, supernatant CXCL13 was detected after lipopolysaccharide treatment in all experiments $(P<0.0048)$, and after tumor necrosis factor- $\alpha$ treatment in one experiment.

\section{Lipopolysaccharide Induces CXCL1 Secretion in Human Endometrial Epithelial Cells In Vitro}

CXCL1 was not detectable in the culture supernatant of the endometrial epithelial cells after interleukin$1 \beta$, interferon- $\gamma$, tumor necrosis factor- $\alpha$ treatment, or control culture in any of five independent experiments (Figure 5b). In contrast, supernatant CXCL1 was detected after lipopolysaccharide treatment in all experiments $(P<0.00023)$.

\section{Discussion}

In addition to stromal plasmacyte infiltration, we confirmed the massive invasion of $\mathrm{B}$ cells into the endometrial functional layer in chronic endometritis. The obvious numerical and topographical differences in B cells from nonpathological endometrium suggest that the identification of this leukocyte subset can be a useful tool for the diagnosis of chronic endometritis. In contrast, other mononuclear leukocytes such as T cells, NK cells, and macrophages did not show distinct differences between chronic endometritis and nonpathological endometrium. These findings were in agreement with one immunohistochemical study, ${ }^{7}$ but not with a flow cytometric study that showed the decrease in NK cell proportion and increase in T-cell proportion in chronic endometritis. ${ }^{28}$ The discrepancy may be due to a difference in methodology. In addition, the number of neutrophils, a hallmark of acute endometritis was similar in the chronic endometritis and nonpathological endometrium. ${ }^{1}$

Of the potential adhesion molecules involved in B-cell extravasation, selectin $\mathrm{E}$ is expressed in the microvessels in chronic endometritis, but not in the nonpathological endometrium. Selectin E is usually undetectable in endothelial cells but appears rapidly after the stimulation with interleukin-1, tumor necrosis factor- $\alpha$, and lipopolysaccharide. ${ }^{29}$ The unique endothelial expression of selectin $E$ in chronic endometritis suggests its role in the initial step of the circulating B-cell extravasation into endometrium.

CXCL13 is one of the most abundant chemokines in endometrial epithelial cells. ${ }^{23}$ Overall, endometrial CXCL13 expression level is upregulated in the women undergoing controlled ovarian hyperstimulation, which is an effective method for growth and ovulation of multiple oocytes but not for successful embryo implantation. ${ }^{30}$ We found that CXCL13 is expressed in the epithelium both in the endometrium with and without chronic endometritis, but its expression in the microvascular endothelium is limited to chronic endometritis. These findings suggest the implication of the endothelial CXCL13 in the selective recruitment of circulating naive $\mathrm{B}$ cells into the endometrium and low embryo implantation rates in chronic endometritis. ${ }^{31}$

We found that lipopolysaccharide enhances selectin E expression and CXCL13 secretion in cultured human uterine microvascular endothelial cells. Lipopolysaccharide is the major component of the outer membrane of Gram-negative bacteria and the ligand to Toll-like receptor 4 expressed on their

Figure 3 Representative microphotographs of immunohistochemistry for chemokines in human endometrium with or without chronic endometritis. The microphotographs represent the immunostaining for CCL2 (a), CCL19 (b), and CXCL8 (c) in chronic endometritis, CXCL13 in the nonpathological endometrium (d) and CXCL13 in chronic endometritis (e), and CXCL1 in the nonpathological endometrium (f) and CXCL1 in chronic endometritis (g). The sections were counterstained with hematoxylin. The arrows indicate the immunostaining with diaminobenzidine. The scale bars indicate $50 \mu \mathrm{m}$. 

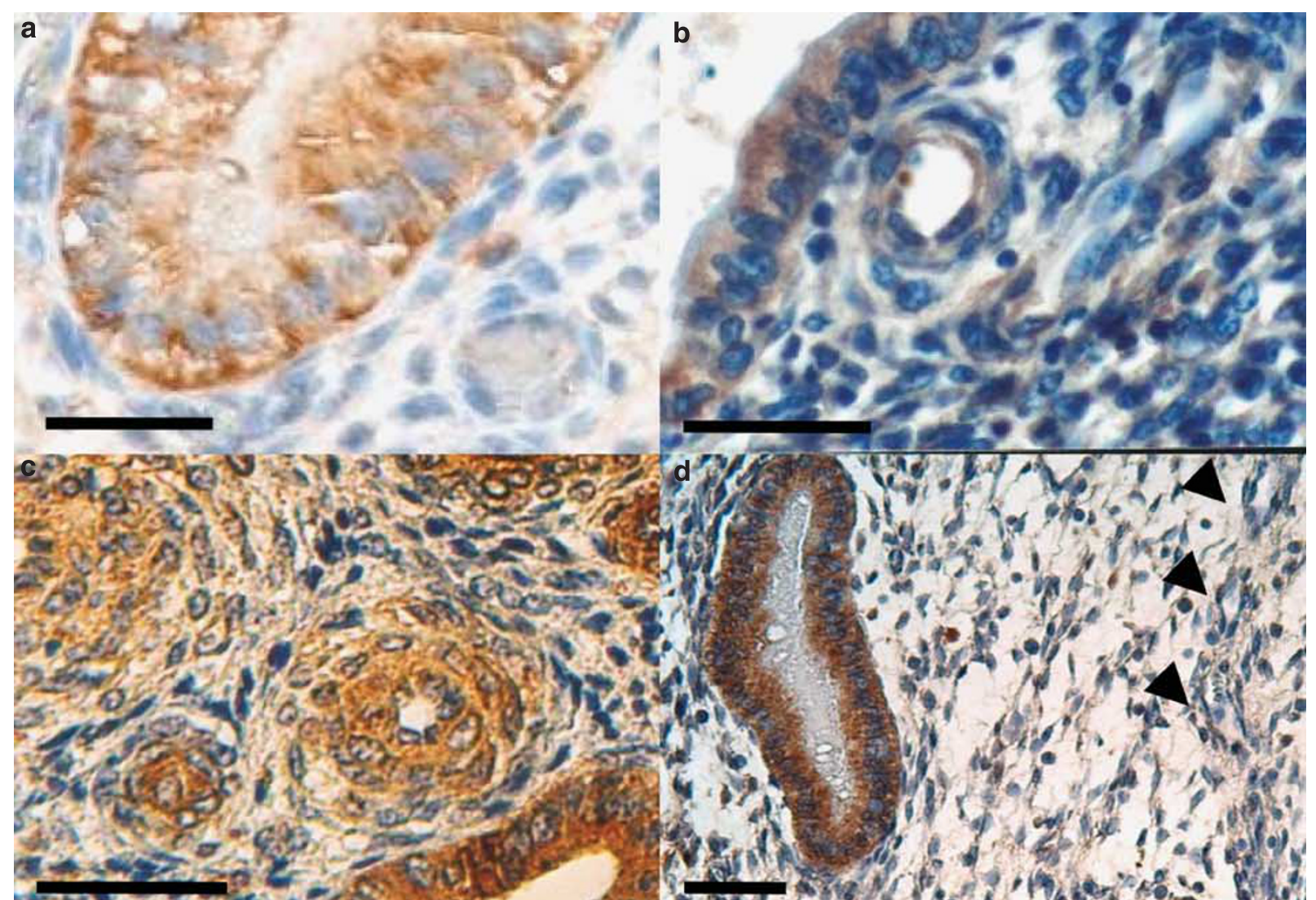

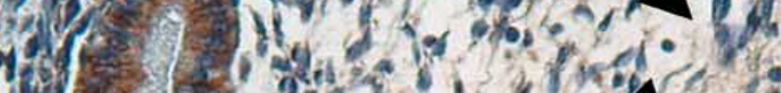

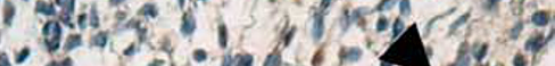
$\because x^{2}=0$ a ith

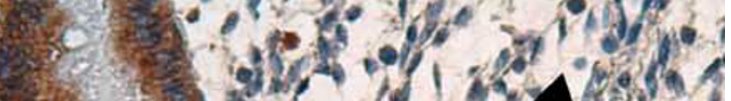

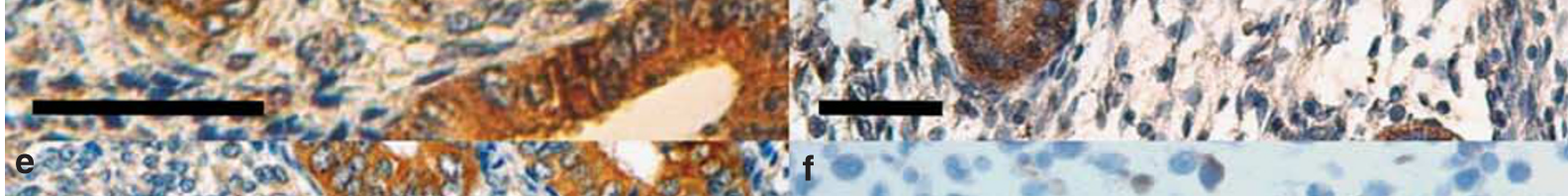

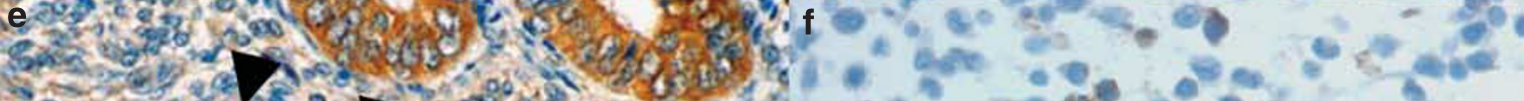

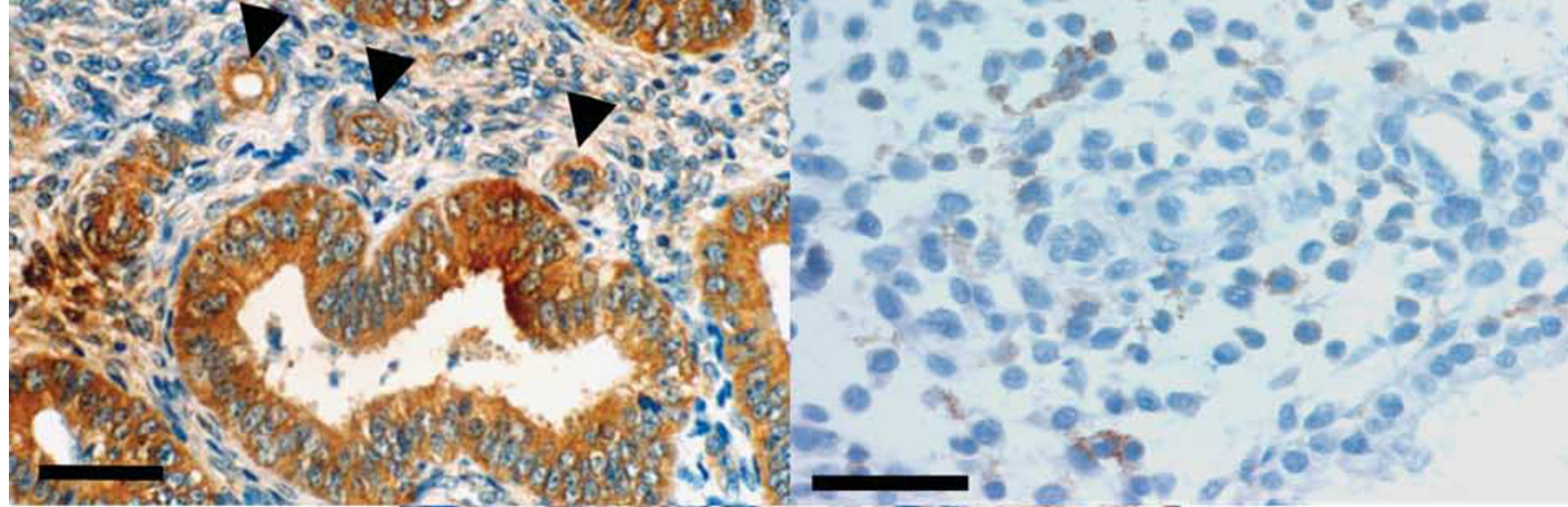

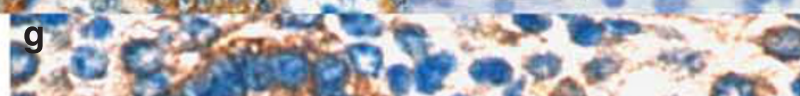

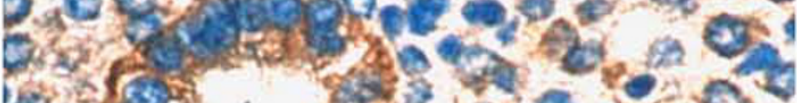

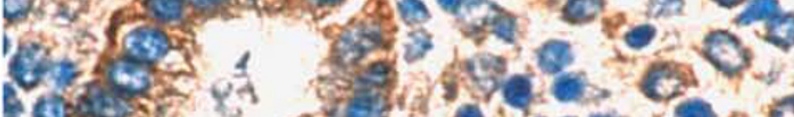

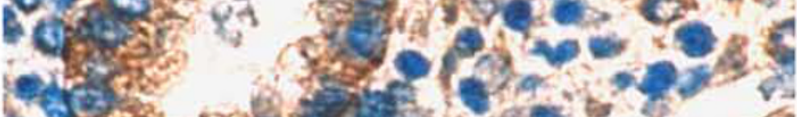

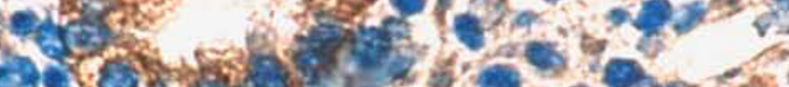

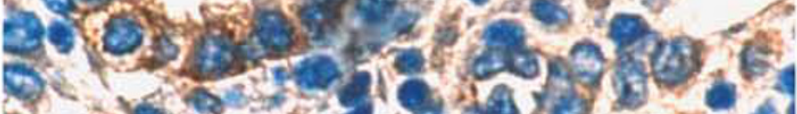
क 2 - to

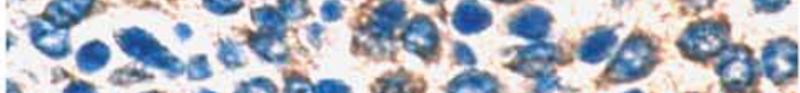

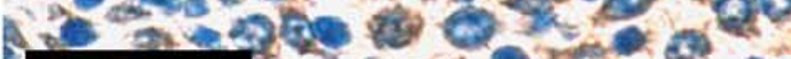



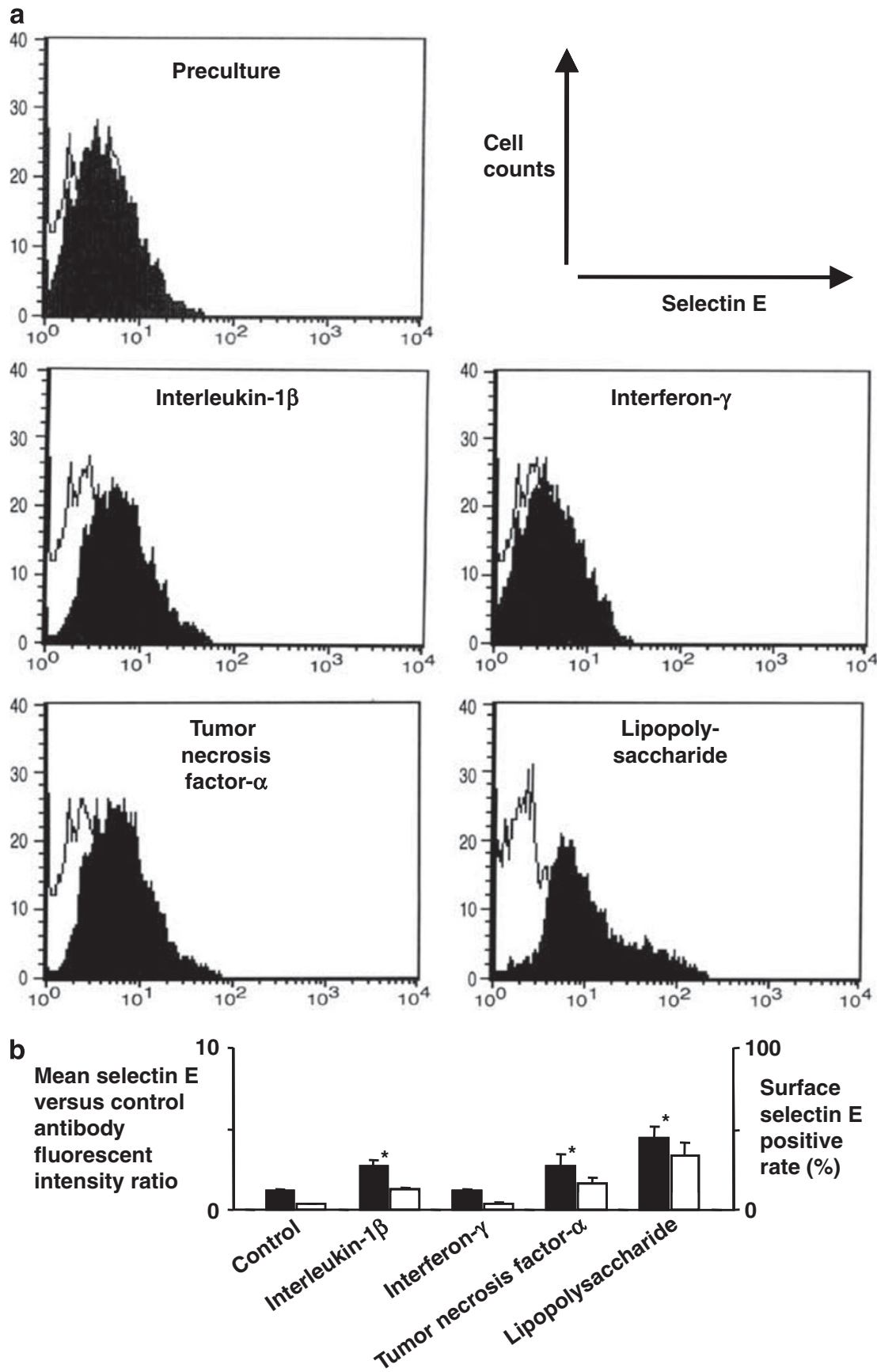

Figure 4 Effect of interleukin-1 $\beta$, interferon- $\gamma$, tumor necrosis factor, and lipopolysaccharide on surface selectin E expression on human uterine microvascular endothelial cells. (a) Representative histograms. The black area indicates the cells immunoreactive for antiselectin E antibody and the white area indicates the cells bound to the control antibody. (b) Summary of five independent experiments.

host cells. ${ }^{32}$ Recent studies demonstrated relatively higher detection rates of a representative anaerobic Gram-negative rod Escherichia coli, than either Chlamydia trachomatis or Ureaplasma urealyticum, in the endometrium with chronic endometritis. ${ }^{33}$ The pathogenicity of Gram-negative rods in chronic endometritis has been supported by the fact that single or combination antibiotic therapies with oral administration of doxycycline, ciprofloxacin, metronidazole, and cefixime for weeks was effective for clearance of stromal plasmacytes and improvement of clinical pregnancy rates. ${ }^{2,34}$ Considering the spectrum of these antimicrobial agents, lipopolysaccharide derived from Gram-negative bacteria may be a candidate mediator of the pathogenesis in chronic endometritis.

CXCL1 is expressed in the endometrial stroma but not in the epithelium under the normal physiological condition. ${ }^{35}$ However, we found that CXCL1 is expressed in both the stroma and epithelium 

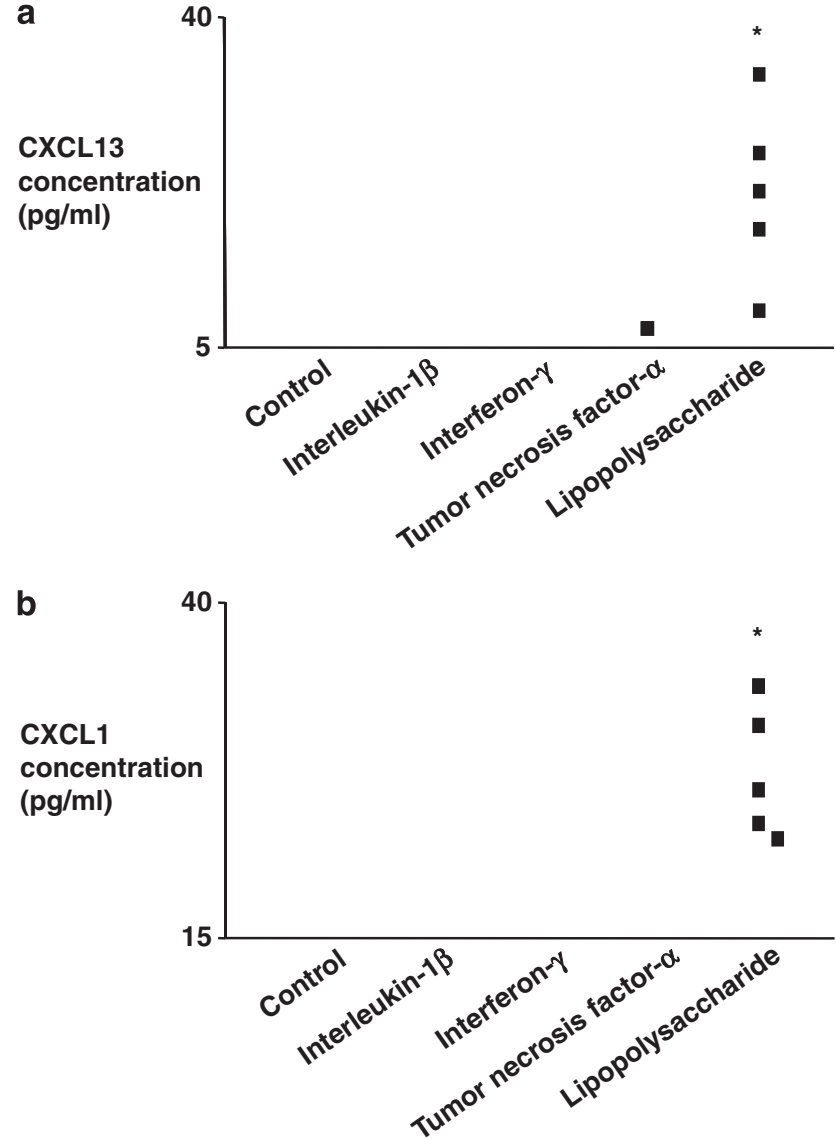

Figure 5 Effect of interleukin- $1 \beta$, interferon- $\gamma$, tumor necrosis factor, and lipopolysaccharide on in vitro CXCL13 secretion by uterine microvascular endothelial cells $(\mathbf{a}, n=5)$ and CXCL1 secretion by endometrial epithelial cells $(\mathbf{b}, n=5)$. The $P$-value in panel (a) is calculated given that the minimal detectable level of CXCL13 was $5 \mathrm{pg} / \mathrm{ml}$ in four experiments in tumor necrosis factor$\alpha$ group. The $P$-value in panel (b) is calculated given that the minimal detectable level of CXCL1 was $15 \mathrm{pg} / \mathrm{ml}$ in five experiments in interleukin- $1 \beta$, interferon- $\gamma$, and tumor necrosis factor- $\alpha$ group.

in chronic endometritis. Lipopolysaccharide can stimulate CXCL1 production by a wide variety of cell types including endothelial cells, macrophages, and fibroblasts. ${ }^{36-38}$ Epithelium-derived CXCL1 in chronic endometritis may have a key role in attracting stromal B cells into glandular epithelium. CXCL1 may also regulate the bioactivity of these plasmacytes by binding to heparan sulfate side chains of proteoglycan syndecan- $1 .^{39}$

In summary, we demonstrated the aberrant expression of the adhesion molecule selectin $\mathrm{E}$ and chemokines CXCL13 and CXCL1 in the endometrium of patients with chronic endometritis, which enables selective B-cell extravasation into endometrial stroma and further into epithelium. This unique microenvironment may be caused by bacterial infection. A fraction of endometrial B cells may undergo in situ differentiation into stroma plasmacytes under these unusual conditions.

\section{Acknowledgement}

This study was supported by a Grant-in-Aid for Scientific Research (no. 16790964) from the Ministry of Education, Culture, Sports, Science and Technology, Japan.

\section{Disclosure/conflict of interest}

The authors declare no conflict of interest.

\section{References}

1 Greenwood SM, Moran JJ. Chronic endometritis: morphologic and clinical observations. Obstet Gynecol 1981;58:176-184.

2 Johnston-MacAnanny EB, Hartnett J, Engmann LL, et al. Chronic endometritis is a frequent finding in women with recurrent implantation failure after in vitro fertilization. Fertil Steril 2010;93:437-441.

3 Ferenczy A. Pathophysiology of endometrial bleeding. Maturitas 2003;45:1-14.

4 PID Evaluation and Clinical Health (PEACH) Study Investigators. Predictors of chronic pelvic pain in an urban population of women with symptoms and signs of pelvic inflammatory disease. Sex Transm Dis 2005; 32:293-299.

5 Crum CP, Egawa K, Fenoglio CM, et al. Chronic endometritis: the role of immunhistochemistry in the detection of plasma cells. Am J Obstet Gynecol 1983; 147:812-815.

6 Yöruköglu K, Kuyucuoglu F. Chronic non-specific endometritis. Gen Diagn Pathol 1998;43:287-290.

7 Disep B, Innes BA, Cochrane HR, et al. Immunohistochemical characterization of endometrial leucocytes in endometritis. Histopathology 2004;45:625-632.

8 Yeaman GR, Guyre PM, Fanger MW, et al. Unique CD8+ T cell-rich lymphoid aggregates in human endometrium. J Leukoc Biol 1997;61:427-435.

9 Kitaya K, Yasuo T. Leukocyte density and composition in human cycling endometrium with uterine fibroids. Hum Immunol 2010;71:158-163.

10 Rosen SD. Ligands for L-selectin: homing, inflammation, and beyond. Annu Rev Immunol 2004;22: 129-156.

11 Rott LS, Briskin MJ, Butcher EC. Expression of $\alpha 4 \beta 7$ and E-selectin ligand by circulating memory $\mathrm{B}$ cells: implications for targeted trafficking to mucosal and systemic sites. J Leukoc Biol 2000;68: 807-814.

12 Kerr SC, Fieger CB, Snapp KR, et al. Endoglycan, a member of the CD34 family of sialomucins, is a ligand for the vascular selectins. J Immunol 2008; 181:1480-1490.

13 Jinquan T, Møller B, Storgaard M, et al. Chemotaxis and IL-8 receptor expression in B cells from normal and HIV-infected subjects. J Immunol 1997; 158:475-484.

14 Kim CH, Broxmeyer HE. Chemokines: signal lamps for trafficking of $\mathrm{T}$ and $\mathrm{B}$ cells for development and effector function. J Leukoc Biol 1999;65:6-15.

15 Alter A, Duddy M, Hebert S, et al. Determinants of human B cell migration across brain endothelial cells. J Immunol 2003;170:4497-4505. 
16 Yamaguchi $\mathrm{T}$, Kitaya $\mathrm{K}$, Daikoku $\mathrm{N}$, et al. Potential selectin L ligands involved in selective recruitment of peripheral blood CD16(-) natural killer cells into human endometrium. Biol Reprod 2006; 74:35-40.

17 Rees MC, Heryet AR, Bicknell R. Immunohistochemical properties of the endothelial cells in the human uterus during the menstrual cycle. Hum Reprod 1993;8:1173-1178.

18 Jones RL, Kelly RW, Critchley HOD. Chemokine and cyclooxygenase-2 expression in human endometrium coincides with leukocyte accumulation. Hum Reprod 1997;12:1300-1306.

19 Nakayama T, Kitaya K, Okubo T, et al. Fluctuation of 6Ckine expression in human endometrium during the menstrual cycle. Fertil Steril 2003;80:1461-1465.

20 Daikoku N, Kitaya K, Nakayama T, et al. Expression of macrophage inflammatory protein- $3 \beta$ in human endometrium throughout the menstrual cycle. Fertil Steril 2004;81(Suppl 1):876-881.

21 Kitaya K, Nakayama T, Daikoku N, et al. Spatial and temporal expression of ligands for CXCR3 and CXCR4 in human endometrium. J Clin Endocrinol Metab 2004;89:2470-2476.

22 Yasuo T, Kitaya K. Effect of ovarian steroids on gene expression profile in human uterine microvascular endothelial cells. Fertil Steril 2009;92:709-721.

23 Dominguez F, Martínez S, Quiñonero A, et al. CXCL10 and IL-6 induce chemotaxis in human trophoblast cell lines. Mol Hum Reprod 2008;14:423-430.

24 Bayer-Garner IB, Korourian S. Plasma cells in chronic endometritis are easily identified when stained with syndecan-1. Mod Pathol 2001;14:877-879.

25 Noyes RW, Hertig AT, Rock J. Dating the endometrial biopsy. Fertil Steril 1950;35:751-754.

26 Kitaya K, Yasuo T. Dermatan sulfate proteoglycan biglycan as a potential selectin L/CD44 ligand involved in selective recruitment of peripheral blood CD16(-) natural killer cells into human endometrium. J Leukoc Biol 2009;85:391-400.

27 Koshiba H, Kitawaki J, Ishihara H, et al. Progesterone inhibition of functional leptin receptor mRNA expression in human endometrium. Mol Hum Reprod 2001; 7:567-572.

28 Matteo M, Cicinelli E, Greco P, et al. Abnormal pattern of lymphocyte subpopulations in the endometrium of infertile women with chronic endometritis. Am J Reprod Immunol 2009;61:322-329.

29 Spertini O, Luscinskas FW, Kansas GS, et al. Leukocyte adhesion molecule-1 (LAM-1, L-selectin) interacts with an inducible endothelial cell ligand to support leukocyte adhesion. J Immunol 1991;147:2565-2573.

30 Macklon NS, van der Gaast MH, Hamilton A, et al. The impact of ovarian stimulation with recombinant FSH in combination with $\mathrm{GnRH}$ antagonist on the endometrial transcriptome in the window of implantation. Reprod Sci 2008;15:357-365.

31 Legler DF, Loetscher M, Roos RS, et al. B cell-attracting chemokine 1, a human CXC chemokine expressed in lymphoid tissues, selectively attracts B lymphocytes via BLR1/CXCR5. J Exp Med 1998;187:655-660.

32 Akira S, Takeda K, Kaisho T. Toll-like receptors: critical proteins linking innate and acquired immunity. Nat Immunol 2001;2:675-680.

33 Cicinelli E, De Ziegler D, Nicoletti R, et al. Chronic endometritis: correlation among hysteroscopic, histologic, and bacteriologic findings in a prospective trial with 2190 consecutive office hysteroscopies. Fertil Steril 2008;89:677-684.

34 Eckert LO, Thwin SS, Hillier SL, et al. The antimicrobial treatment of subacute endometritis: a proof of concept study. Am J Obstet Gynecol 2004;190: 305-313.

35 Nasu K, Fujisawa K, Arima K, et al. Expression and regulation of growth-regulated oncogene- $\alpha$ in human endometrial stromal cells. Mol Hum Reprod 2001;7: 741-746.

36 Wen DZ, Rowland A, Derynck R. Expression and secretion of gro/MGSA by stimulated human endothelial cells. EMBO J 1989;8:1761-1766.

37 Becker S, Quay J, Koren HS, et al. Constitutive and stimulated MCP-1, GRO- $\alpha$, $-\beta$, and $-\gamma$ expression in human airway epithelium and bronchoalveolar macrophages. Am J Physiol 1994;266:278-286.

38 Lin M, Carlson E, Diaconu E, et al. CXCL1/KC and CXCL5/LIX are selectively produced by corneal fibroblasts and mediate neutrophil infiltration to the corneal stroma in LPS keratitis. J Leukoc Biol 2007;81: 786-792.

39 Wang D, Sai J, Richmond A. Cell surface heparan sulfate participates in CXCL1-induced signaling. Biochemistry 2003;42:1071-1077. 